\title{
Una propuesta metodológica para el análisis melódico de enunciados orales espontáneos con partículas modales del alemán ${ }^{1}$
}

\author{
José TORREGROSA AZOR \\ Universidad de Barcelona \\ jtorregrosa@ub.edu
}

Recibido: 9 de diciembre de 2013

Aceptado: 18 de febrero de 2014

\begin{abstract}
RESUMEN
En este trabajo se examina la relación que se establece entre entonación y partículas modales del alemán. Para ello, en la primera sección se estudia la bibliografía especializada que trata este tema específico, tomando como punto de partida la tesis de Kriwonossow de 1977. Observamos que esta relación ha sido escasamente desarrollada desde un punto de vista objetivo e instrumental. En la segunda sección, describimos un método para analizar la entonación y su relación con las partículas modales del alemán. Por una parte, el método de análisis descrito posibilita analizar enunciados orales reales, genuinos y espontáneos emitidos por múltiples informantes; y, por otra parte, permite obtener contornos entonativos normalizados y comparables con el objetivo de establecer las diferentes modalidades fonológicas del alemán y definir el significado pragmático de las partículas modales del alemán.
\end{abstract}

Palabras clave: Entonación, partículas modales del alemán, análisis melódico del habla.

\section{A Methodological Proposal for the Melodic Analysis of Spontaneous Spoken Utterances with German Modal Particles}

\begin{abstract}
In the present paper we analyse the relationship between intonation and German modal particles. In section 1., we review the literature on this specific topic, since Kriwonossow wrote his dissertation in 1977 and we observe that this relationship has been an aspect hardly developed from an objective and instrumental point of view. In section 2., we describe a method for analysing intonation and its relation to German modal particles. The method enables, on the one hand, to analyse real and spontaneous spoken utterances produced by German multiple subjects and, on the other hand, to obtain standardised intonation patterns for establishing phonological modalities in German and define the pragmatic meaning of German modal particles.
\end{abstract}

Keywords: Intonation, German Modal Particles, Melodic Analysis of Speech.

SUMARIO: 1. Introducción. 2. Entonación y partículas modales del alemán. 3. Análisis melódico del habla. 3.1. Procedimiento analítico. 3.2. Desarrollo analítico. 4. Conclusión y futuras investigaciones.

\footnotetext{
${ }^{1}$ Este trabajo se inscribe en el proyecto de investigación financiado por el Ministerio de Economía y Competitividad Análisis Melódico del Habla y Modelos Didácticos, nº de ref. FFI2013-41915-P.
} 


\section{Introducción}

El presente artículo forma parte de una investigación más amplia que tiene por objeto presentar y exponer el método de Análisis Melódico del Habla (AMH) descrito en Cantero (2002) e implementado posteriormente en Cantero / Font-Rotchés (2009), a través del cual es posible analizar y describir las características melódicas de la lengua alemana, los patrones entonativos de las diferentes modalidades y los correspondientes márgenes de dispersión o variantes entonativas relevantes para la determinación del significado pragmático.

El método AMH ha sido aplicado con éxito en numerosas investigaciones que estudian la entonación del castellano (cfr. Cantero / Font-Rotchés 2007, Ballesteros 2011, Font-Rotchés / Mateo 2013), del catalán ( $c f r$. Font-Rotchés 2007, FontRotchés 2011), del italiano (Devís 2011) y del portugués de Brasil (Fonseca 2013), pero también en lenguas pertenecientes a familias lingüísticas diferentes como el húngaro (Baditzné 2012), el chino (Liu 2003), el sueco (Martorell 2010) y el alemán ( $c f r$. Torregrosa 2010, Torregrosa 2013, Torregrosa / Font-Rotchés en prensa).

El objetivo principal consiste en analizar las características melódicas de enunciados que contienen partículas modales del alemán (PM) a partir de producciones orales, genuinas y espontáneas realizadas por un número amplio y diverso de informantes cuya lengua materna es el alemán.

Teniendo en cuenta que la investigación que nos proponemos llevar a cabo se encuentra actualmente en curso, en el presente trabajo exponemos, en primer lugar, el estado de la cuestión relativo a la relación existente entre entonación y PM $\mathrm{y}$, en segundo lugar, se centra la atención en la descripción detallada del método analítico utilizado.

\section{Entonación y partículas modales del alemán}

Las PM es un campo de investigación que experimenta un desarrollo inusitado a partir de la década de los sesenta del siglo veinte. El interés por estudiar las PM viene promovido por un cambio de orientación en la Lingüística, desde un punto de vista teórico y también desde un punto de vista metodológico ( $c f r$. Helbig 1988). El foco de atención se desplaza desde el análisis lingüístico de la lengua como sistema para interesarse por el uso oral, especialmente por las funciones comunicativas que desempeña y por los diferentes significados pragmáticos que se transmiten ligados a los diferentes contextos y situaciones comunicativas.

En primer lugar, conviene hacer referencia a los trabajos pioneros de Kriwonossow (1977) y de Weydt (1969) que estudian las PM desde esta nueva perspectiva. Se trata de dos referentes fundamentales que ordenan el panorama científico en torno a las partículas en general, pero especialmente en relación a las PM que habían sido notoriamente denostadas anteriormente por investigadores adscritos a la estilística normativa, cuyas contribuciones promovían prescindir de su uso porque se consideraban palabras carentes de sentido y de funcionalidad, utilizando 
diversas designaciones terminológicas para referirse a las PM que, en general, presentan connotaciones negativas (cfr. von der Gabelentz 1901, Reiners 1961, Lindqvist 1961, Thiel 1962, Adler 1964, Schröder 1965, Colditz 1966, entre otros). Ambas obras representan los cimientos teóricos y metodológicos sobre los cuales se fundamenta la investigación que se desarrolla con posterioridad en relación a las PM.

El objetivo que se propone Kriwonossow es, por una parte, delimitar el grupo de las PM con respecto a las demás categorías que se caracterizan por el rasgo "inflexibilidad" y, por otra parte, establecer su distribución sintáctica y determinar las funciones que desempeñan en la lengua alemana así como los diferentes matices de significado pragmático que aportan a la frase (Kriwonossow 1977: 45-46).

Para ello se fundamenta en el estado de confianza que le permite el dominio intuitivo e inconsciente de la lengua alemana (Kriwonossow 1977: 47), cuyas unidades de análisis -las PM- son objeto de investigación. Esta perspectiva le permite realizar el análisis distribucional de las partículas modales y del contexto oracional en que se manifiestan, a partir del significado comunicativo y pragmático que se vehicula.

Para Kriwonossow (1977: 307), la entonación es el mecanismo principal que posee la lengua oral a través del cual se transmite el "significado modalsubjetivo", esto es, la valoración personal e intencionalidad comunicativa que el hablante expresa a través del contenido proposicional que es portador del "significado modal-objetivo". Asimismo, las PM, que conjuntamente con la entonación forman una entidad fonética independiente, se consideran igualmente formas de expresión de la lengua oral, a través de las cuales no solo se comunica el significado modal-subjetivo, sino que además actúan como instrumentos de mediación entre la lengua oral y la lengua escrita. Este es el caso, por ejemplo, de la manifestación de PM en textos escritos que simulan el ámbito oral de la lengua, como en los pasajes dialogados, monólogos, etc. En ellos las PM representan un papel muy importante, ya que en este caso son el único medio a través del cual se hace posible la manifestación del significado modal-subjetivo, del valor añadido que el hablante imprime a través de los enunciados (Kriwonossow 1977: 309). Sin embargo, conviene indicar un pequeño matiz que, aunque no parezca ser relevante para un hablante nativo que presenta un dominio intuitivo e inconsciente de la lengua, desde el punto de vista del alemán como lengua extranjera resulta de gran importancia: el lector no nativo que se enfrenta con este tipo de texto probablemente no sería capaz, solo a partir de la lectura, de aplicar convenientemente los patrones entonativos que requeriría la lectura en alemán ni de extraer el significado pragmático que se intenta transmitir a partir del texto escrito.

Así, el corpus de datos en que se basa para realizar el análisis distribucional de las PM consiste en ejemplos tomados de obras literarias (Kriwonossow 1977: 330), así como también de conversaciones orales, en los cuales tendrá especial consideración el análisis de la percepción melódica de cada enunciado. Este será un importante aspecto metodológico discutido en Lütten (1977: 108-111). En pri- 
mer lugar, la autora cuestiona que la estructura entonativa que se desprende de la lectura de textos literarios pueda ser considerada como equiparable a la del habla espontánea. En segundo lugar, considera que si para Kriwonossow el principal medio de expresión del significado modal-subjetivo en lengua oral se realiza a través de la entonación, y que las PM son un medio adicional a través del cual también se puede transmitir el significado modal-subjetivo, el ámbito de uso real de las partículas modales no corresponde entonces al habla espontánea, sino a la lengua escrita, o mejor dicho, a textos literarios que presumiblemente adoptan las formas auténticas del habla espontánea o que estilísticamente son equiparables a estas. Sin embargo, las reflexiones que a este respecto se plantea Lütten han sido escasamente consideradas por las investigaciones que se han sucedido hasta la actualidad (cfr. Heggelund 2001) y las pocas que tratan la relación entonación-PM -al menos todas aquellas que han sido consultadas para la presente investigaciónadoptan un enfoque metodológico si no idéntico, muy similar al que propone Kriwonossow ( $c f r$. Vural 2000 como ejemplo paradigmático).

El trabajo de Weydt (1969) no es especialmente relevante para la cuestión que tratamos en este artículo, la relación entre entonación y PM, pero es sumamente influyente para el desarrollo posterior de estudios y análisis contrastivos entre PM de la lengua alemana y sus correspondencias lingüísticas en otras lenguas ${ }^{2}$.

En general, se observa que, por una parte, el corpus de datos que se emplea para realizar el análisis de la entonación procede generalmente de fuentes escritas de diverso género, pero extraído principalmente de obras literarias. Por este motivo, la lectura individual de los enunciados, sea mentalmente o en voz alta, es el procedimiento habitual para determinar las características acústicas de las $\mathrm{PM}^{3}$ y del

\footnotetext{
${ }^{2}$ A continuación, presentamos algunos ejemplos de estudios contrastivos entre las PM y sus posibles equivalentes lingüísticos en otras lenguas pertenecientes a familias lingüísticas diferentes. Con la lengua inglesa Schubiger (1965), AsbaCh-SchnitKer (1977), O’SUllivan / RöSler (1989) y NEHLS (1989); con la lengua neerlandesa WeSTHEIDE (1989) y FoOLEN (2003); con la lengua sueca HEINRICHS (1981) y AIJMER (1996); con la lengua noruega HEGGELUND (2001); con la lengua islandesa BADE (2013). Con la lengua francesa WEYDT (1969) y DALMAS (1989); con la lengua castellana BEerbom (1991); con la lengua portuguesa HARdEn (1989), Franco (1989), SCHMIDT-RAdEFELDT (1989), KunOw (1997), NunEs (2012) y AQUINo (2012); con la lengua rumana UlRICH (1989) y STĂNESCU (1989). Con la lengua serbocroata LiČEN (1989); con la lengua checa NEKULA (1996); con la lengua eslovena KoROŠEC (1999); con la lengua polaca KATNY (1989). Con la lengua japonesa SEKIGUCHI (1977), KAWASHIMA (1989), KoSAKA (1989) y WERNER (1998); con la lengua coreana CHOI (1989). Con la lengua turca Vural (2000). Con la lengua finesa LiEFLÄNDER-KoISTINEN (1989).

${ }^{3}$ Creemos conveniente indicar que un aspecto ampliamente consensuado por el que generalmente se caracterizan las PM hace referencia al rasgo [-tónico]. Cfr. ARNDT (1960: 330); KRIWONOSSOW (1977: 240 y 311-312); WEYDT (1969: 25 y 68); BuBLITZ (1978: 37); GORNIK-GERHARDT (1981: 33 y 72); Bastert (1985: 38); Helbig (1988: 32); Hentschel (1986: 155); Hentschel / WeydT (1989:14); Wegener (1989: 57); StǍnescu (1989: 268); BEerbom (1991: 27); Meibauer (1994: 224); NeKula (1996: 46 y 62); HegGelund (2001: 1); GirÁldez (2002: 56); FoOlen (2003); y KWON (2005: 11-12), entre otros. Sin embargo, mientras que unos investigadores establecen claramente una relación directa entre PM y el rasgo [-tónico], otros investigadores admiten variantes
} 
contorno melódico del enunciado en el que se emiten. Si las circunstancias lo permiten, los datos resultantes del análisis pueden ser contrastados con las emisiones o percepciones individuales realizadas por otros informantes ( $c f r$. Kriwonossow 1977, Vural 2000, Kwon 2005, Ikoma 2007 y Moroni 2010, entre otros). Es decir, solo a través de la competencia lingüística en alemán sería posible efectuar el análisis de la entonación y la relación que se establece con las PM, lo cual delimita o circunscribe su estudio y análisis exclusivamente al ámbito lingüístico germánico (Steinmüller 1981: 139). Finalmente, la descripción del contorno melódico se representa numéricamente o mediante la gradación "alto", "medio" y "bajo" dependiendo del nivel tonal que se intuye (cfr. Kriwonossow 1977, Pusch 1981 y Vural 2000) o a través de la transcripción verbal o fonética que adoptaría la forma de la curva melódica ( $c f r$. Schubiger 1965 y Özen 1989) y de la cual dependerá la determinación del tipo de modalidad oracional, que se indica gráficamente mediante los signos “.”, “?”, “!”, “...”, etc.

Sin embargo, el ámbito de uso predominante en el que suelen emitirse las PM es la lengua oral y especialmente en un registro coloquial y familiar, como se indica en Ferrer (2000: 253):

Die deutschen MPn werden in erster Linie der mündlichen, insbesondere auch der Umgangssprache zugeschrieben. Das bedeutet aber nicht, dass sie nicht auch in geschriebenen Texten z.B. bestimmten Texten vorkommen, ihre Domäne ist aber v.a. das Gespräch in seinen vielfältigen Formen, wobei die Gesprächpartner durch die Verwendung von MPn ihre Einstellungen dem Gesagten gegenüber zum Ausdruck bringen. (Ferrer 2000: 253)

La mayoría de los autores se esfuerzan por justificar la validez de los análisis de tipo acústico que realizan sobre un corpus de datos escrito.

A partir de esta perspectiva se determinan las características suprasegmentales de las PM. Por ejemplo, en Gornik-Gerhardt (1981) se establecen hasta seis posibles significados distintos de la PM schon a partir de la interpretación intuitiva de la melodía de enunciados escritos: "einschränkende Bestätigung", "abgeschwächter Widerspruch", "negative Beurteilung", "plausible Begründung", "Dringlichkeit" y "a fortiori Argumentation". Asimismo, siguiendo este procedimiento se determina la modalidad oracional de los enunciados (cfr. Ferrer 1999 y 2010) y la asociación de determinadas PM a determinadas modalidades oracionales: partículas declarativas, partículas imperativas y partículas interrogativas (cfr. Franck 1980, Bastert 1985, Nekula 1996, Graefen 2000 y Kwon 2005, entre otros).

En Franck (1980: 188) la emisión de la PM doch se asocia con la modalidad imperativa con una entonación de tipo "drängender Ton". En Kwon (2005: 36-37) la interpretación melódica de enunciados declarativos con la PM ja exige un determinado tipo de entonación que depende del contexto comunicativo en el cual se

semánticas de algunas PM que se caracterizan por el rasgo [+tónico] (cfr. GORNIK-GERHARDT 1981: 33 y 72, MEIBAUER 1994: 224, HeNTSCHEL 1986:155, DOHERTY 1987: 128-138). 
emite. Así, en el enunciado “Ach, guten Morgen, Gerda! Da bist du ja endlich!", el emisor ha tenido que "entonarlo" como una exclamación: "[...] Man kann sich aber leicht vorstellen, dass der Sprecher diesen Satz intonatorisch als Ausruf geäussert hat" (Kwon 2005: 36-37). Otro caso es el de atribuir directamente las características melódicas de un enunciado a partir de la PM que aparece. En Bastert (1985: 72) se indica que la partícula modal doch puede ser enunciada en oraciones de tipo interrogativo aunque con ciertas restricciones; a saber: "DOCH kann nur in Fragen mit Aussage-Wortstellung und Frageintonation stehen, [...]" (Bastert 1985: 72), lo que se ejemplifica a través del enunciado "wir kennen uns doch, nicht wahr?".

Lo cierto es que, desde un punto de vista no nativo, no sabríamos, en primer lugar, a qué tipo de entonación interrogativa se refiere, si con final ascendente o con final descendente; si la melodía del enunciado es más o menos enfática, o si presenta mayor o menor contraste tonal entre los segmentos tonales que lo integran. Es decir, no sabríamos cómo "entonar" este enunciado o al que alude Kwon, o cómo "entonar" en alemán un enunciado emitido con "drängender Ton", si no hacemos un uso intuitivo y arbitrario de nuestro propio inventario entonativo en nuestra lengua nativa; esto es, emitiendo este enunciado alemán con entonación castellana o en cualquier otra lengua diferente a la alemana.

Otra serie de ejemplos, en este caso aludiendo a diferentes patrones melódicos paralingüísticos, se pueden encontrar en Graefen (2000), donde se indica que la frase "Paß doch auf!" se pronuncia con una "entonación de indignación"; o esta otra frase "Hör doch endlich auf!", que se pronuncia con una "entonación de enfado, contrariedad o disgusto'; o esta otra "Das ist doch klasse!", que se entona como una 'exclamación de entusiasmo'; y, finalmente, esta otra frase "Das konnte ich doch nicht wissen!", que se pronuncia con una "entonación en defensa ante un reproche".

Pero..., ¿cómo se emite, en alemán, una entonación de indignación; o de enfado, contrariedad o disgusto; o una exclamación de entusiasmo; o en defensa ante un reproche? La interpretación melódica de los enunciados con PM que ofrecen los investigadores que tratan la relación entonación-PM desde un plano perceptivo de tipo intuitivo genera un panorama extremadamente complejo.

Creemos que tales interpretaciones solo pueden ser sustentadas y justificadas a partir de un método de análisis objetivo e instrumental de la materia fónica. La determinación de las características melódicas del enunciado o de cada uno de los grupos fónicos se debería establecer en base a un corpus de datos orales genuinos, reales y espontáneos; es decir, a partir de la materia fónica de las emisiones de enunciados pronunciados en situaciones comunicativas reales y cotidianas; y no de forma intuitiva a partir de un corpus de datos escritos. 


\section{Análisis melódico del habla}

En esta sección presentamos el método de Análisis Melódico del Habla (AMH) (cfr. Cantero 2002 y Cantero / Font-Rotchés 2009). En primer lugar, mostramos en el apartado 3.1. el procedimiento analítico que permite segmentar las unidades del habla basándose en un corpus de enunciados reales y espontáneos emitidos por informantes diversos en cuanto a edad y género, a partir de los cuales es posible obtener contornos entonativos generalizables y comparables.

En segundo lugar, se desarrolla en el apartado 3.2. el procedimiento analítico descrito en el apartado 3.1. Para ejemplificar el desarrollo metodológico, se han seleccionado algunos enunciados del corpus que utilizaremos en nuestra investigación.

\subsection{Procedimiento analítico}

El método de Análisis Melódico del Habla (AMH) desarrollado en Cantero (2002) y sistematizado en Cantero / Font-Rotchés (2009) es un procedimiento de análisis de la entonación que permite obtener de los enunciados una representación melódica generalizable, teniendo en cuenta la información fónica relevante contenida en los mismos enunciados y discriminando aquella que no lo es. Este método de análisis está basado en el principio de "jerarquía fónica", según el cual los sonidos del habla no son una mera sucesión de emisiones, sino que se organizan en bloques fónicos, el núcleo de los cuales es un segmento vocálico (Cantero 2002: 147). Desde esta perspectiva, los elementos de la cadena fónica se encuentran perfectamente estructurados y jerarquizados alrededor de los sonidos vocálicos, que son los elementos que aportan información melódica relevante, cumpliendo una función integradora dentro de la estructura discursiva.

Por tanto, la unidad de análisis es el segmento tonal, el sonido vocálico, ya que es el elemento de la cadena fónica que tiene tono y del cual se puede obtener la frecuencia fundamental (F0). En torno al segmento vocálico se estructuran los demás elementos de la cadena fónica. Así, el procedimiento que se sigue consiste en obtener y anotar los valores frecuenciales absolutos $(\mathrm{Hz})$ de cada uno de los sonidos vocálicos de que se compone el enunciado. El gráfico que resulta después de procesar los datos se genera a partir de las variaciones frecuenciales de los segmentos vocálicos y muestra la abstracción de la melodía del enunciado analizado.

Sin embargo, el contorno que se representa conserva todavía los rasgos fónicos individuales de cada informante, de cuyo análisis no es posible aún extraer generalizaciones comparables. Es necesario, por tanto, obtener configuraciones que describan únicamente la estructura melódica de los enunciados, prescindiendo de las características fónicas individuales, para que los contornos resultantes puedan ser comparables con otras copias melódicas producidas por cualquier otro informante y poder extraer y estudiar los rasgos melódicos comunes en una determinada variedad lingüística. Por este motivo, no resulta suficiente la obtención del valor frecuencial de los segmentos vocálicos, sino que es necesario llevar a cabo un proceso de relativización de estos datos, que consiste en transformar los valores 
frecuenciales en datos porcentuales que reflejan la relación tonal existente entre un valor frecuencial absoluto y el valor inmediatamente anterior. Finalmente, se realiza un proceso de normalización, a través del cual se estandarizan estos valores tomando como dato de referencia inicial el número 100, al cual se aplican los porcentajes obtenidos en el proceso de relativización.

A continuación, presentamos en primer lugar la fórmula para realizar la relativización de los valores frecuenciales y, en segundo lugar, la fórmula para estandarizar o normalizar los valores relativos:

Fórmula de relativización:

Fórmula de estandarización:

$$
\begin{aligned}
& \mathrm{V}_{\mathrm{RELn}+1}=\left[\left(\mathrm{V}_{\mathrm{ABSn}+1}-\mathrm{V}_{\mathrm{ABSn}}\right) / \mathrm{V}_{\mathrm{ABSn}}\right] * 100 \\
& \mathrm{~V}_{\mathrm{Sn}+1}=\mathrm{V}_{\mathrm{Sn}}+\left[\left(\mathrm{V}_{\mathrm{RELn}+1} * \mathrm{~V}_{\mathrm{Sn}}\right) / 100\right]
\end{aligned}
$$

La variable $\mathrm{V}_{\mathrm{ABS} \text { n }}$ corresponde al valor absoluto del segmento vocálico de referencia. $\mathrm{V}_{\mathrm{ABS} \mathrm{n}+1}$ es el valor frecuencial del segmento vocálico que sucede al valor de referencia y del cual se determina la diferencia relativa en \% con respecto al segmento vocálico precedente. $\mathrm{V}_{\mathrm{REL} n+1} \mathrm{o}$ valor relativo expresa la diferencia melódica porcentual entre el valor absoluto $\mathrm{V}_{\mathrm{ABS} n+1} \mathrm{y}$ el valor inmediatamente anterior que actúa como valor de referencia. Finalmente, $\mathrm{V}_{\mathrm{S} n+1}$ corresponde al valor estandarizado de $V_{\text {REL } n+1}$ con respecto a $V_{S}$. Este último es el valor estándar que actúa como valor de referencia. A partir del algoritmo resultante se representa gráficamente el contorno entonativo normalizado.

La lógica del cálculo de las fórmulas expresa la progresión ordenada de las sucesivas variaciones tonales que se dan entre los segmentos vocálicos contiguos de la cadena fónica y define la estructura melódica de los enunciados analizados.

Aplicando el procedimiento descrito se genera la curva melódica estándar, objetiva, comparable y generalizable desde un punto de vista lingüístico. El gráfico resultante no se corresponde exactamente con la curva melódica pronunciada por el emisor, pero sí que es semejante, ya que de esta forma se centra la atención en los aspectos estrictamente tonales y posibilita que pueda ser comparado tonalmente con otras copias estandarizadas con independencia de las características fónicas de los informantes que los han emitido.

\subsection{Desarrollo analítico}

En esta sección mostraremos con ejemplos el desarrollo del proceso analítico presentado en la sección 3.1. El primer paso consiste en obtener un corpus de enunciados que permita analizar la relación entre entonación y PM en contextos comunicativos reales y que sean producciones orales que contengan PM realizadas por un número de informantes muy amplio y heterogéneo.

Una vez que se establezca el corpus de datos, el siguiente paso es realizar el análisis melódico a través de una metodología que permita analizar los datos de una forma objetiva mediante instrumentos adecuados para el tratamiento de la señal acústica y que los resultados obtenidos puedan ser comparables y generalizables. 
Actualmente, el corpus de datos que utilizaremos para llevar a cabo la investigación consta de 802 emisiones producidas por 140 informantes diferentes obtenidas a partir de la grabación en vídeo de diversos canales y programas de la televisión alemana, así como también de sondeos de opinión grabados en la calle y en diversas localidades alemanas. Algunos de estos enunciados han sido utilizados en este trabajo para ejemplificar el proceso analítico del método que utilizaremos.

La diferencia principal de nuestro corpus de datos orales con respecto a otros corpus a través de los cuales se analiza la relación entonación-PM consiste en que no procede de fuentes escritas. Por tanto, no se ha obtenido a partir de frases escritas en obras literarias u otro tipo de fuente escrita, sea en forma de papel o en forma digitalizada de transcripciones verbales. El corpus tampoco procede de frases preparadas por el investigador para ser leídas y grabadas posteriormente en un laboratorio, sino que, como ya hemos indicado, procede de manifestaciones orales reales del habla alemana producidas por diversos informantes en contextos comunicativos de tipo informal o coloquial.

Así, los enunciados proceden de grabaciones de 11 programas de la televisión alemana y de una serie de 16 entrevistas en las calles de diversas localidades alemanas. Los programas de televisión corresponden a los géneros "Talk show", "Live talk", magacines y debates, el $50 \%$ de los cuales ha sido obtenido de programas del género "Talk show". Este tipo de programas, así como también los sondeos de opinión en la calle, son especialmente aptos para un tipo de investigación en el que se analice la entonación a partir de un registro lingüístico natural y cotidiano, como el que se propone en este trabajo, porque ofrecen la ventaja de poder obtener emisiones orales de un número de informantes muy amplio y heterogéneo, tanto en las variables de edad como de sexo, de formación, de profesión, etc., garantizando de esta forma la ausencia de emisiones producidas por personal especializado como por ejemplo lingüistas, actores, escritores o locutores de radio. Otra ventaja que ofrece este tipo de programas es que en su desarrollo se puede apreciar una amplia gama de contextos comunicativos de tipo informal, aspecto que resulta especialmente interesante para el análisis de las PM.

Los informantes cubren un amplio espectro social. E1 56\% de los informantes son mujeres y el $44 \%$ restante son hombres. La franja de edad de los informantes se establece entre los 16 y los 77 años. Por lo que se refiere al origen o procedencia, el $78 \%$ de los informantes es originario de la República Federal de Alemania y tiene residencia habitual en este país en el momento de las grabaciones. De este grupo, el $49 \%$ es originario o reside habitualmente en el tercio septentrional de Alemania; el $28 \%$ es originario o reside habitualmente en la zona central de Alemania; y, finalmente, el $23 \%$ es originario o reside de forma habitual en el tercio meridional de Alemania. El 7\% de los informantes procede de otras zonas dentro del ámbito lingüístico de la lengua alemana, situadas en la zona sur y este de Austria (Kärnten, Steiermark y Wien) y al norte de Suiza. Finalmente, se desconoce objetivamente la procedencia y lugar de residencia del $15 \%$ de los informantes, aunque el dominio de la lengua objeto de análisis corresponde a un nivel nativo. 
En relación al grado de formación, el $35 \%$ de los informantes ha cursado estudios superiores, el $38 \%$ estudios secundarios y el $27 \%$ ha finalizado estudios primarios.

Cada uno de los enunciados seleccionados se etiqueta convenientemente para su posterior localización y digitalización y se realiza el análisis melódico mediante el programa Praat ${ }^{4}$ aplicando el método de Análisis Melódico del Habla descrito en el apartado 3.1.

Anteriormente, hemos indicado que la unidad mínima de análisis es el segmento tonal que se compone de, al menos, un sonido vocálico. Por tanto, el primer paso a seguir consiste en obtener los valores frecuenciales de los segmentos vocálicos de cada uno de los enunciados. Para llevar a cabo esta operación, se crea una tabla en Excel como la que se muestra en la figura 1 para anotar cada uno de los segmentos tonales que componen cada enunciado y su correspondiente valor frecuencial o absoluto obtenido a través del aplicativo Praat.

\begin{tabular}{|l|l|l|l|}
\hline Segmentos & $\mathrm{Hz}$ & Porcentajes & Curva estándar \\
\hline & & & \\
\hline & & & \\
\hline & & & \\
\hline & & & \\
\hline & & & \\
\hline & & & \\
\hline & & & \\
\hline & & & \\
\hline & & & \\
\hline & & & \\
\hline & & & \\
\hline
\end{tabular}

Figura 1. Tabla de anotación

A continuación, se extraen los valores frecuenciales (F0) de cada segmento tonal de las muestras seleccionadas y digitalizadas con la extensión WAV o NSP. Por defecto, el programa presenta en la parte superior de la ventana de análisis el oscilograma del enunciado y la localización de los pulsos glotales procesados en la muestra y, en la parte inferior de la ventana, el espectrograma, que nos permite localizar fácilmente los segmentos vocálicos, y también una representación de las variaciones tonales en forma de contorno no estilizado.

Para obtener los valores frecuenciales (F0) de cada segmento tonal, debemos atender principalmente a los datos acústicos mostrados en la mitad inferior de la ventana de Praat, donde aparece el espectrograma de la muestra que se analiza y, por tanto, los formantes de los sonidos vocálicos -las zonas más oscuras-, que son las unidades que informan de la frecuencia fundamental (F0).

La figura 2. expone el aspecto que hemos descrito, tomando como ejemplo el enunciado etiquetado como I-ZH2-19 "Wie kommt es ei(ge)ntlich dazu?"

\footnotetext{
${ }^{4}$ http://www.praat.org.
} 


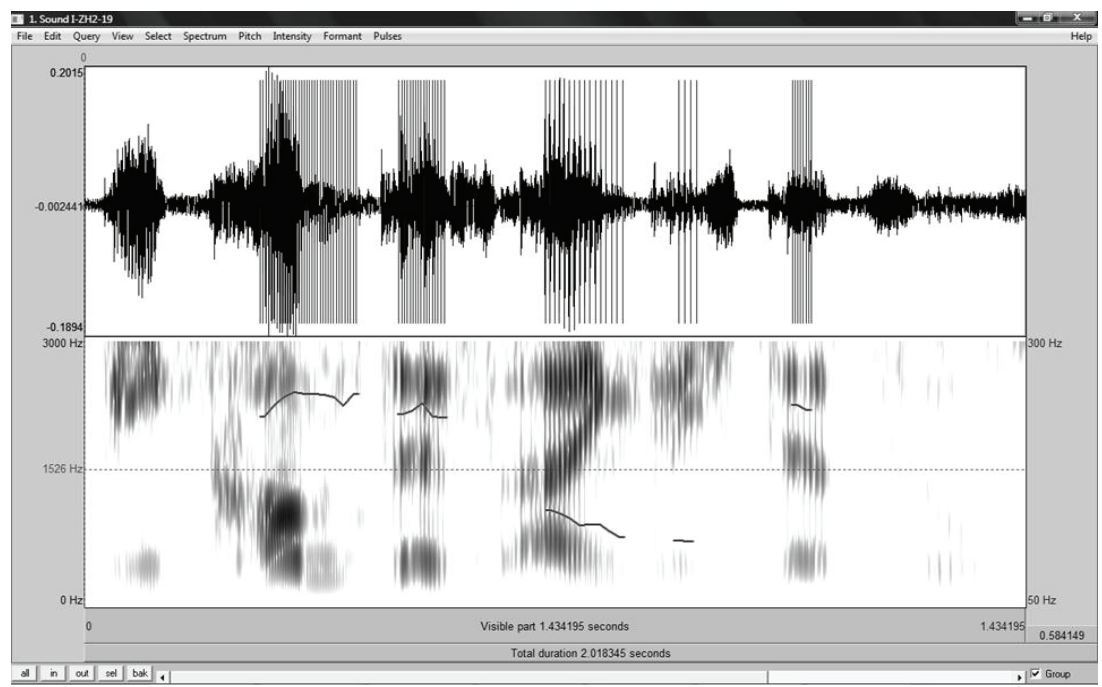

Figura 2. Enunciado I-ZH2-19

La figura 3 muestra la selección de cada uno de los segmentos tonales que componen el enunciado I-SJ1-53 "Warum könnte er denn hier sein?". El valor frecuencial de cada segmento tonal, esto es, de cada selección que se ha realizado, se indica en el margen derecho y será el valor que trasladaremos a la tabla de anotación de la figura 1. La selección del segmento tonal requiere que sea precisa, para lo cual el investigador no solo deberá tener en cuenta las zonas sombreadas del espectrograma, sino también otros aspectos acústicos importantes (cfr. FontRotchés 2007 y Cantero / Mateo 2011).

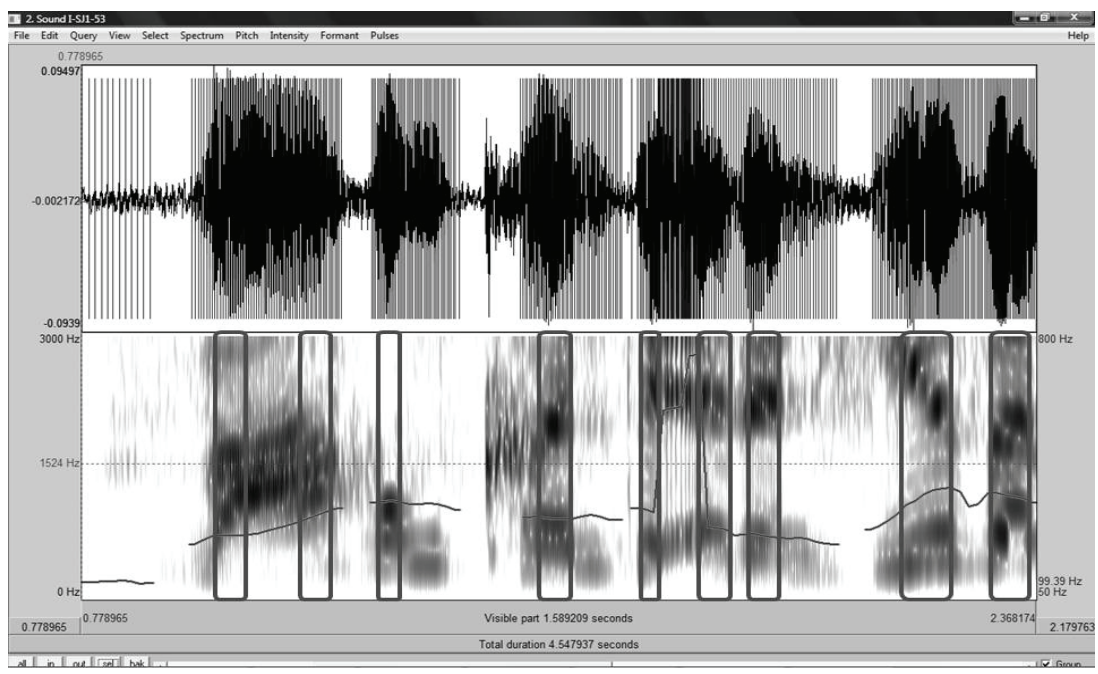

Figura 3. Selección de segmentos tonales en el enunciado I-SJI-53 
Después de haber obtenido todos los valores frecuenciales de los segmentos tonales de cada enunciado, se trasladan a la tabla de anotación para poder calcular posteriormente los valores relativos y los valores estándar. En la figura 4 mostramos la tabla de anotación después de haber trasladado los valores frecuenciales de cada segmento tonal obtenidos a través del aplicativo Praat.

\begin{tabular}{|l|c|l|l|}
\hline Segmentos & $\mathrm{Hz}$ & Porcentajes & Curva estándar \\
\hline Wa & 227 & & \\
\hline Wa* & 302 & & \\
\hline rum & 325 & & \\
\hline könn & 278 & & \\
\hline te & 296 & & \\
\hline er & 288 & & \\
\hline er* & 242 & & \\
\hline denn & 229 & & \\
\hline hier & 268 & & \\
\hline hier* & 365 & & \\
\hline sein & 340 & & \\
\hline
\end{tabular}

Figura 4. Segmentos tonales y valores absolutos (F0) del enunciado I-SJ1-53

Los elementos marcados con un asterisco indican que en la emisión del segmento tonal se ha producido una inflexión tonal superior al $10 \%$ que consideramos relevante desde un punto de vista melódico, no tratándose en ningún caso de una repetición. Por este motivo, se han obtenido dos valores frecuenciales diferentes para el mismo segmento tonal, el valor inicial y el valor final. Del algoritmo resultante podemos representar gráficamente el contorno del enunciado I-SJ1-53. Sin embargo, esta curva melódica todavía conserva las variables de tipo micromelódico propias del informante que ha emitido el enunciado. Estas variables de tipo fisiológico son únicas para cada informante y, por tanto, no son comparables a otras copias del mismo enunciado pronunciadas por otros informantes.

En el caso del enunciado I-SJ1-53, las variables micromelódicas corresponden a un informante femenino dentro de una franja de edad de entre 25 y 35 años. Por una parte, sabemos a través de una simple prueba perceptiva o a través de un análisis acústico instrumental que la voz femenina se caracteriza generalmente por una frecuencia superior a la voz masculina y, por otra parte, que la configuración anatómica de los resonadores supraglóticos encargados de amplificar las frecuencias producidas en los pliegues vocales es única para cada persona. Por tanto, solo a través de los valores frecuenciales obtenidos de cada segmento tonal no es posible obtener un contorno melódico que pueda ser comparable con otras copias melódicas de características similares y emitidas por otros informantes. En la figura 5 mostramos la curva resultante que representa la sucesión ordenada de las variaciones frecuenciales para el enunciado I-SJ1-53. 


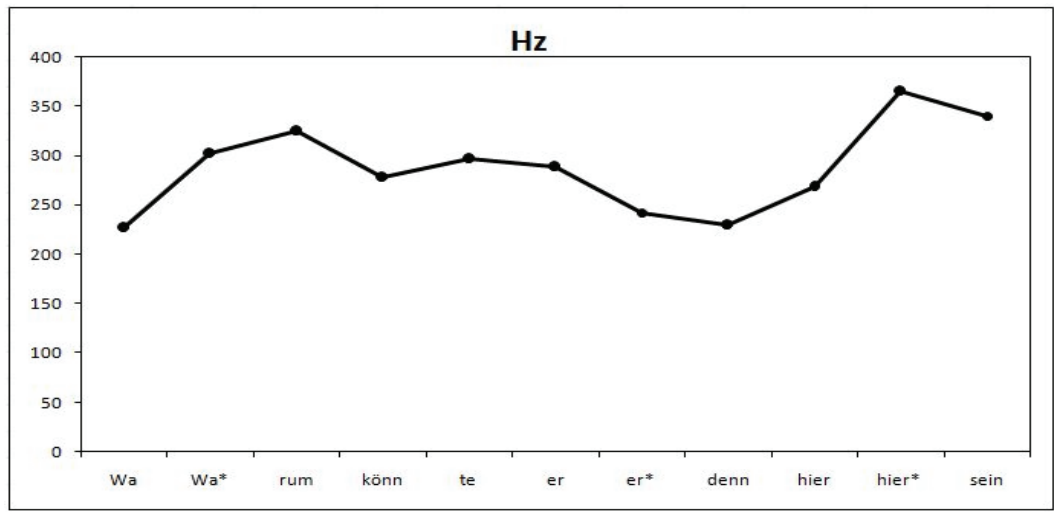

Figura 5. Contorno frecuencial del enunciado I-SJ1-53

Para poder obtener contornos melódicos de los enunciados que puedan compararse con otras copias melódicas es necesario transformar los valores absolutos mediante un proceso de relativización, donde la información relevante no reside en el valor mismo de cada uno de los segmentos tonales, sino que reside en la distancia tonal existente entre ellos. Para obtener los valores relativos de los valores frecuenciales es preciso aplicar la fórmula de relativización. El resultado que se obtiene indica la distancia tonal entre segmentos representada en forma de porcentajes y que se incluyen en la columna correspondiente de la tabla de anotación. La figura 6 muestra los segmentos tonales del enunciado I-SJ1-53 y los correspondientes valores frecuenciales y normalizados.

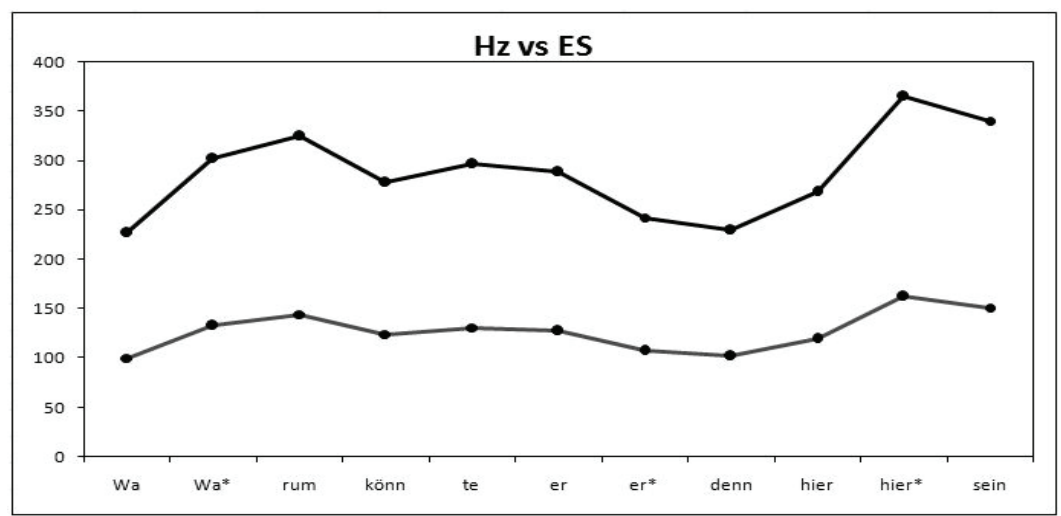

Figura 6. Valores absolutos (F0) y estándar (ES) de los segmentos tonales del enunciado I-SJ1-53

Como hemos indicado anteriormente, los valores relativos reflejan la distancia tonal entre los segmentos tonales y se presentan en forma de porcentaje positivo, si la distancia tonal de un segmento es superior al valor frecuencial del segmento inmediatamente anterior, $\mathrm{y}$ en forma de porcentaje negativo, si la distancia tonal es 
inferior al valor frecuencial del segmento tonal precedente. A partir de los valores relativos que se obtienen, se puede generar la curva estándar mediante el procedimiento de normalización. Para obtener los valores estandarizados, se aplica la fórmula de estandarización descrita partiendo de un valor de referencia que hemos establecido en 100. El algoritmo resultante permite generar la curva melódica estándar que, ahora sí, es comparable con otras copias melódicas y, por tanto, generalizable.

\begin{tabular}{|l|c|c|c|}
\hline Segmentos & $\mathrm{Hz}$ & Porcentajes & Curva estándar \\
\hline Wa & 227 & $100 \%$ & 100 \\
\hline Wa* & 302 & $33 \%$ & 133 \\
\hline rum & 325 & $8 \%$ & 143 \\
\hline könn & 278 & $-14 \%$ & 122 \\
\hline te & 296 & $6 \%$ & 130 \\
\hline er & 288 & $-3 \%$ & 127 \\
\hline er* & 242 & $-16 \%$ & 107 \\
\hline denn & 229 & $-5 \%$ & 101 \\
\hline hier & 268 & $17 \%$ & 118 \\
\hline hier* & 365 & $36 \%$ & 161 \\
\hline sein & 340 & $-7 \%$ & 150 \\
\hline
\end{tabular}

Figura 7. Tabla de anotación completa

La figura 7 muestra la tabla de anotación completa con todos los datos para generar la representación abstracta de la entonación del enunciado, es decir, la curva melódica estándar.

En la figura 8, la curva estándar describe un contorno similar al descrito a partir de los valores frecuenciales, pero solo es similar en la forma. El dato principal y representativo en la curva frecuencial es precisamente el valor frecuencial y las variaciones que describe el contorno son diferencias frecuenciales entre segmentos. Sin embargo, en la curva estándar la información relevante es la distancia tonal entre segmentos y no únicamente el valor tonal.

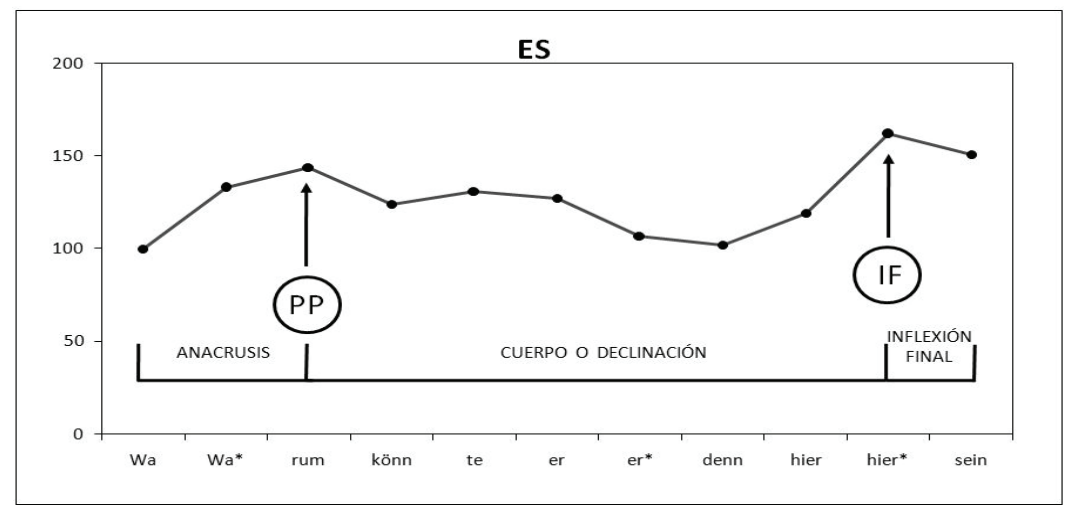

Figura 8. Características de los elementos funcionales del contorno 
Para poder realizar la comparación entre contornos y la caracterización fonética y fonológica es necesario atender a las diferentes partes que componen el contorno: el anacrusis, el cuerpo del contorno o declinación y la inflexión final. El anacrusis está constituido por todos los segmentos tonales que preceden al primer segmento tónico. Por su parte, la inflexión final (IF) la conforman todos los segmentos tonales emitidos a partir de la última vocal tónica. Finalmente, la declinación o cuerpo del contorno está formado por los segmentos tonales comprendidos entre el primer segmento tónico o primer pico (PP) y el último segmento tónico, a partir del cual se desarrolla la IF.

Además de estos tres rasgos melódicos principales presentados anteriormente, Cantero (2002: 160-161) menciona otros dos rasgos melódicos más, el campo tonal y el registro tonal, que pueden ser determinantes para la caracterización fonológica de un contorno, es decir, la determinación de los patrones entonativos de una lengua y los diferentes márgenes de dispersión.

El método AMH permite establecer la estructura melódica de una lengua e interpretarla formalmente a diferentes niveles de descripción lingüística: a nivel prelingüístico, a nivel lingüístico y a nivel paralingüístico.

Según Cantero / Font-Rotchés, la entonación prelingüística "[...] constituye el elemento integrador del habla, el principio unificador del discurso, que permite su inteligibilidad, cuya organización caracteriza los distintos dialectos (...), y cuya desorganización afecta al habla de los extranjeros [...]" (Cantero / Font-Rotchés 2007: 69). El análisis de la entonación prelingüística permite determinar la estructura acentual de una lengua, en este caso las características melódicas de la lengua alemana, y culmina con el desarrollo de la fase acústica del método de AMH descrita anteriormente, que consiste en la obtención de contornos melódicos estandarizados a partir de producciones orales espontáneas; esto es, contornos estilizados que contienen toda la información melódica relevante y desprovistos de las variaciones micromelódicas, para que puedan ser comparados con otras copias estilizadas.

El análisis de la entonación lingüística se desarrolla a partir de la fase acústica y consiste en establecer y caracterizar fonológicamente los diferentes patrones entonativos de una lengua mediante pruebas perceptivas con informantes nativos. La IF es el elemento funcional del contorno que aporta la información melódica relevante y que permite describir y diferenciar formalmente los contornos según las características fonológicas / \pm interrogación/, / \pm enfático/ y / \pm suspendido/. Así, a partir de la caracterización fonológica de los contornos entonativos será posible determinar las diferentes realizaciones entonativas de las modalidades oracionales.

Finalmente, siguiendo la terminología que se propone en Cantero / FontRotchés (2007), el análisis de la entonación paralingüística permite establecer y caracterizar los diferentes márgenes de dispersión o alocontornos de cada uno de los patrones entonativos, a través de las cuales se transmiten significados de carácter expresivo. Así, en este nivel de análisis será posible determinar cómo se entona en alemán la indignación, el enfado, la contrariedad, el disgusto, el entusiasmo e incluso cómo se entona la defensa ante un reproche. 


\section{Conclusión y futuras investigaciones}

Teniendo en cuenta los resultados obtenidos en estudios preliminares que analizan la relación que se establece entre entonación y PM (cfr. Torregrosa 2010, Torregrosa 2013), la metodología que hemos presentado y desarrollado en el presente trabajo es un procedimiento instrumental fiable y adecuado para el análisis de las características melódicas de las PM a partir de un corpus de datos obtenido de emisiones espontáneas realizadas por múltiples informantes, ya que permite obtener conclusiones objetivas, generalizables y comparables.

El análisis de la relación entre entonación y PM debería fundamentarse en un corpus de datos procedentes de producciones orales reales y espontáneas y atender a los diversos medios de expresión de la oralidad, entre los cuales forman parte no solo las características específicas de la lengua oral sin mediación escrita, sino también las características melódicas, entonativas y pragmáticas de los enunciados en los que se emiten PM.

Como futuras investigaciones propondríamos incrementar sustancialmente el corpus de datos y describir, en primer lugar, cómo se organizan melódicamente las unidades lingüísticas del discurso para determinar el perfil acentual, rítmico y melódico del alemán en habla espontánea y las características melódicas de las PM. En segundo lugar, caracterizaríamos los tonemas o unidades fonológicas con el objetivo de definir los rasgos distintivos de las diferentes modalidades oracionales y su asociación con determinadas PM. Finalmente, a partir de la caracterización melódica y fonológica sería posible determinar y establecer los diferentes márgenes de dispersión o variantes expresivas de las unidades fonológicas y establecer el tipo de significado pragmático que se vehicula a través de la emisión de las PM.

\section{Referencias bibliográficas}

ADLER, H. G., «Füllwörter», Muttersprache 74 (1964), 52-55.

AIJMER, K., «Swedish modal particles in a contrastive perspective», Language Sciences 18 (1996), 393-427.

AQUINO, M., A função dinâmica das partículas modais alemãs doch e ja no ensino de línguas. Memoria de Máster. Brasil: Universidade Federal de Minas Gerais 2012. http://hdl.handle.net/1843/LETR-8ST23Y. [07/12/2013].

ARNDT, W., «'Modal particles' in Russian and German», Word 16 (1960), 323-336.

ASBACH-SCHNITKER, B., «Die Satzpartikel wohl. Eine Untersuchung ihrer Verwendungsbedingungen im Deutschen und ihrer Wiedergabemöglichkeiten im Englischen», en: WEYDT, H. (ed.), Aspekte der Modalpartikeln: Studien zur deutschen Abtönung. Tübingen: Niemeyer. [Konzepte der Sprach- und Literaturwissenschaft, 23] 1977, 38-61.

BADE, S., Das kannst $d u$ doch nicht einfach sagen! Eine kontrastive, übersetzungsorientierte Analyse deutscher Abtönungspartikeln und ihrer isländischen Entsprechungen. Memoria de Máster. Universidad de Islandia 2013. http://hdl.handle.net/1946/16508. [01/12/2013]. 
BADITZNÉ, K., Spanish intonation of Hungarian learners of Spanish: yes-or-no questions. Tesis Doctoral. Budapest: University of Eötvös Loránd. Biblioteca Phonica 15 (2012). http://www.ub.edu/lfa. [01/10/2014].

BAllesteros, M. P., La entonación del español del norte. Tesis Doctoral. Universidad de Barcelona 2011. http://hdl.handle.net/10803/109963. [09/03/2014].

BASTERT, U., Modalpartikel und Lexikographie: eine exemplarische Studie zur Darstellbarkeit von "doch” im einsprachigen Wörterbuch. Tübingen: Niemeyer [Reihe germanistische Linguistik, 58] 1985.

BEERBOM, CH., Modalpartikeln als Übersetzungsproblem. Eine kontrastive Studie zum Sprachenpaar Deutsch-Spanisch. Tesis Doctoral publicada en 1992 en Frankfurt am Main [etc.]: Peter Lang [Heidelberger Beiträge zur Romanistik, 26] 1991.

Bublitz, W., Ausdrucksweisen der Sprechereinstellung im Deutschen und Englischen. Untersuchungen zur Syntax, Semantik und Pragmatik der deutschen Modalpartikeln und Vergewisserungsfragen und ihrer englischen Entsprechungen. Tübingen: Niemeyer [Linguistische Arbeiten, 57] 1978.

CANTERO, F. J., Teoría y análisis de la entonación. Barcelona: Edicions de la Universitat de Barcelona 2002.

CANTERO, F. J. / FONT-RotCHÉS, D., «Entonación del español peninsular en habla espontánea: patrones melódicos y márgenes de dispersión». Moenia 13 (2007), 69-92.

CANTERo, F. J. / FonT-Rotchés, D., «Protocolo para el análisis melódico del habla». Estudios de Fonética Experimental (ELE) 18 (2009), 17-32.

CANTERo, F. J. / MATEO, M., «Análisis Melódico del Habla: complejidad y entonación en el discurso». Oralia 14 (2011), 105-127.

CHOI, K-R., «Schon und noch und ihre koreanischen Entsprechungen imi und ajik», en: Weydt, H. (ed.), Sprechen mit Partikeln. Berlín: Walter de Gruyter 1989, 129-140.

COLDITZ, S., «Einige Wörter in ihrer vielseitigen Verwendbarkeit», Deutsch als Fremdsprache (DaF) 3(1) (1966), 49-52.

DALMAS, M., «Sprechakte vergleichen: ein Beitrag zur deutsch französischen Partikelforschung», en: WEYDT, H. (ed.), Sprechen mit Partikeln. Berlín: Walter de Gruyter 1989, 228-239.

Devís, E., «La entonación del español hablado por italianos». Didáctica (Lengua y Literatura) (2011), 35-38.

DoHERTY, M., Epistemic meaning. Berlín / Heidelberg: Springer-Verlag. [Springer series in language and communication, 21] 1987.

FERRER, H., «El modo oracional interrogativo en la lingüística germánica: rasgos formales y función, taxonomías y subtipos», Revista de Filología Alemana (RdFA) 7 (1999), 239-271. http://revistas.ucm.es/index.php/RFAL/article/view/RFAL9999110239A. [07/12/2013].

FERRER, H., «Auf der Suche nach spanischen Modalpartikeln: pero und pues als pragmatische Konnektoren. Stand der kontrastiven Partikelforschung DeutschSpanisch», Revista de Filología Alemana (RdFA) 8 (2000), 253-271. http://revistas.ucm.es/index.php/RFAL/article/view/RFAL0000110253A. [07/12/2013].

FERRER, H., «Criterios para el estudio de la modalidad en alemán y en español: los modos oracionales y las palabras modales». Revista de Filología Alemana Anejo II (2010), 8198. http://revistas.ucm.es/index.php/RFAL/article/view/36534. [07/12/2013].

FONSECA, A., Caracterización de la entonación del español hablado por brasileños. Tesis Doctoral. Universidad de Barcelona 2013. http://hdl.handle.net/10803/134929. [09/03/2014]. 
FonT-RotchÉS, D., L'entonació del català. Barcelona: Publicacions de l'Abadia de Montserrat 2007.

FONT-RotCHÉS, D., «Èmfasis bàsics de l'entonació en els contorns del català central», en: LLORET, M-R / C. PONS (eds.), Noves aproximacions a la fonología i la morfología del català. Valencia: Institut Interuniversitari de Filología Valenciana 2011, 193-216.

FONT-RotchÉS, D. / MATEO, M., «Entonación de las interrogativas absolutas del español meridional en habla espontánea», Onomázein 28 (2013), 256-275.

FoOLEN, A., «Niederländisch toch und Deutsch doch: Gleich oder doch nicht ganz?», Linguistik Online 13/1 (2003). http://www.linguistik-online.de/13_01/foolen_a.html. [07/12/2013].

FrancK, D., Grammatik und Interaktion. Stilistische Pragmatik des Dialogs und die Bedeutung deutscher Modalpartikeln. Königstein/Taunus: Scriptor. [Referencia en Bastert 1985] 1980.

FrANCO, A., «Modalpartikeln im Portugiesischen - Kontrastive Syntax, Semantik und Pragmatik der portugiesischen Modalpartikeln», en: WEYDT, H. (ed.), Sprechen mit Partikeln. Berlín: Walter de Gruyter 1989, 240-255.

GIRÁlDEZ, O., Valor ilocutivo de las partículas modales alemanas y su aplicación a la didáctica del alemán como lengua extranjera: Una propuesta metodológica. Tesis Doctoral. Universidad de Sevilla 2002. http:/fondosdigitales.us.es/tesis/resultadosbusqueda/?authors in=125. [08/12/2013].

GORNIK-GERHARDT, H., $\mathrm{Zu}$ den Funktionen der Modalpartikel 'schon' und einiger ihrer Substituentia. Tübingen: Narr [Tübinger Beiträge zur Linguistik, 155] 1981.

Graefen, G., «Ein Beitrag zur Partikelanalyse - Beispiel: doch», Linguistik Online 6 (2000). http://www.linguistik-online.de/2_00/graefen_a.html. [08/12/2013].

HARDEN, TH., «Na ja - ein vielseitiger Kommentar und seine portugiesischen Entsprechungen», en: WEYDT, H. (ed.), Sprechen mit Partikeln. Berlín: Walter de Gruyter 1989, 141-149.

HegGelund, K. T., «Zur Bedeutung der deutschen Modalpartikeln in Gesprächen unter besonderer Berücksichtigung der Sprechakttheorie und der Daf-Perspektive», Linguistik Online 9 (2001). http://www.linguistik-online.de/9_01/Heggelund_a.html. [07/12/2013].

HEINRICHS, W., Die Modalpartikeln im Deutschen und Schwedischen. Eine kontrastive Analyse. Tübingen: Niemeyer. [Linguistische Arbeiten, 101] 1981.

HelBIG, G., Lexikon deutscher Partikeln. 3a ed. revisada, 1994. Berlín: Langenscheidt Verlag Enzyklopädie VEB 1988.

Hentschel, E., Funktion und Geschichte deutscher Partikeln. Ja, doch, halt und eben. Tübingen: Niemeyer. [Germanistische Linguistik, 63] 1986.

Hentschel, E. / Weydt, H., «Wortartenprobleme bei Partikeln», en: WeydT, H. (ed.), Sprechen mit Partikeln. Berlín: Walter de Gruyter 1989, 3-18.

IKOMA, M., Prosodische Eigenschaften der deutschen Modalpartikeln. Hamburg: Verlag Dr. Kovač [PHILOLOGIA - Sprachwissenschaftliche Forschungsergebnisse, Bd. 103] 2007.

KATNY, A., «Wohl als Partikel und Modalwort und seine Übersetzungsmöglichkeiten ins Polnische», en: WEYDT, H. (ed.), Sprechen mit Partikeln. Berlín: Walter de Gruyter 1989, 196-203.

KaWASHIMA, A., «Textsorte und partikeln im Japanischen und Deutschen», en: WeYDT, H. (ed.), Sprechen mit Partikeln. Berlín: Walter de Gruyter 1989, 276-281. 
KOROŠEC, V., Der Gebrauch von Abtönungspartikeln bei den slowenischen Schülern. Tesina de licenciatura. Pedagošca Fakulteta Maribor (Facultad de Pedagogía de Maribor) 1999.

KosAKA, K., «Abtönungspartikel denn und Satznominalisierung», en: WEYDT, H. (ed.), Sprechen mit Partikeln. Berlín: Walter de Gruyter 1989, 160-170.

Kriwonossow, A., Die modalen Partikeln in der deutschen Gegenwartsprache. Göppingen: Kümmerle. [Göppinger Arbeiten zur Germanistik, 214] [1963] 1977.

KunOw, I., Diskurspartikeln im Portugiesischen. Gesprächsanalytische Studien zur Abtönung und Redeorganisation in informeller und institutioneller Kommunikation. Tesis Doctoral. Universität Freiburg 1997. http://d-nb.info/964681587. [07/12/2013].

KWON, M-J., Modalpartikeln und Satzmodus. Untersuchungen zur Syntax, Semantik und Pragmatik der deutschen Modalpartikeln. Tesis Doctoral. Ludwig-MaximiliansUniversität Múnich 2005. http://d-nb.info/978873653. [07/12/2013].

LIČEN, M., «Die serbokroatische Partikel 'PA' und ihre deutschen Entsprechungen», en: WEydT, H. (ed.), Sprechen mit Partikeln. Berlín: Walter de Gruyter 1989, 171-184.

LIEFLÄNDER-KOISTINEN, L., «Zum deutschen doch und finnischen - han. Beobachtungen zur Übersetzbarkeit der deutschen Abtönungspartikel», en: WEYDT, H. (ed.), Sprechen mit Partikeln. Berlín: Walter de Gruyter 1989, 185-195.

LINDQVIST, A., Satzwörter. Eine vergleichende syntaktische Studie. Göteborg: Acta Universitatis Gothoburgensis 1961.

LiU, Y-H., La entonación del español hablado por taiwaneses. Tesis Doctoral. Universidad de Barcelona 2003. Biblioteca Phonica 2. http://www.ub.edu/lfa. [09/05/2014].

LÜTTEN, J., Untersuchungen zur Leistung der Partikeln in der gesprochenen deutschen Sprache. Philosophischen Fakultät der Wilhelms-Universität Münster. Tesis Doctoral publicada. Göppingen: Kümmerle [Göppinger Arbeiten zur Germanistik, 218] 1977.

MARTORELL, L., Les interrogatives absolutes de l'espanyol parlat per suecs. Memoria de Máster. Universidad de Barcelona 2010. http://hdl.handle.net/2445/32302. [12/06/2014].

MeIBAuer, J., Modaler Kontrast und konzeptuelle Verschiebung: Studien zur Syntax und Semantik deutscher Modalpartikeln. Tübingen: Niemeyer 1994.

Moroni, M. C., Modalpartikeln zwischen Syntax, Prosodie und Informationsstruktur. Frankfurt am Main: Peter Lang 2010.

NeHLS, D., «German modal particles rendered by english auxiliary verbs», en: WEYDT, H. (ed.), Sprechen mit Partikeln. Berlín: Walter de Gruyter 1989, 282-292.

NeKUlA, M., System der Partikeln im Deutschen und Tschechischen: unter besonderer Berücksichtigung der Abtönungspartikeln. Tübingen: Niemeyer. [Linguistische Arbeiten, 355] 1996.

NUNES, E. C. R., As partículas modais da língua alemã. Um estudo com base nos contos "Nachts schlafen die Ratten doch" de Borchert e "Berlin Bolero" de Schulze. Memoria de Máster. Brasil: Universidade Federal de Santa Catarina 2012. http://repositorio.ufsc.br/xmlui/handle/123456789/91055. [07/12/2013].

O’Sullivan, E. / RÖSLER, D., «Wie kommen Abtönungspartikeln in deutsche Übersetzungen von Texten, deren Ausgangssprachen für diese keine direkten Äquivalente haben?», en: WEYDT, H. (ed.), Sprechen mit Partikeln. Berlín: Walter de Gruyter 1989, 204-216.

ÖZEN, E., «Anforderungen an ein 'Partikelwörterbuch für Deutschlerner'», en: WEYDT, H. (ed.), Sprechen mit Partikeln. Berlín: de Gruyter 1989, 634-644.

Pusch, L. F., «Ganz», en: WEYDT, H. (ed.), Partikeln und Deutschunterricht. Abtönungspartikeln für Lerner des Deutschen. Heidelberg: Groos 1981, 31-43. 
REINERS, L., Stilkunst. Ein Lehrbuch deutscher Prosa. Múnich: Beck 1961.

SCHMIDT-RADEFELDT, J., «Partikeln und Interaktion im deutschportugiesischen Sprachvergleich», en: WEYDT, H. (ed.), Sprechen mit Partikeln. Berlín: Walter de Gruyter 1989, 256-266.

SCHRÖDER, G., «Zu einigen bedeutungsgeminderten Adverbien (Würzwörter)», Deutsch als Fremdsprache (DaF) 2(1) (1965), 31-33.

SCHUBIGER, M., «English intonation and German modal particles: a comparative study», Phonetica 12 (1965), 65-84.

SEKIGUCHI, T., «Was heißt doch?», en: WeYDT, H. (ed.), Aspekte der Modalpartikeln: Studien zur deutschen Abtönung. Tübingen: Niemeyer. [Konzepte der Sprach- und Literaturwissenschaft, 23] 1977, 19-37.

STĂNESCU, S., «Zum Status der Partikeln im Deutschen und im Rumänischen», en: WEYDT, H. (ed.), Sprechen mit Partikeln. Berlín: Walter de Gruyter 1989, 267-275.

STEINMÜLLER, U., «Akzeptabilität und Verständlichkeit. Zum Partikelgebrauch von Ausländern», en: WeYDT, H. (ed.), Partikeln und Deutschunterricht. Abtönungspartikeln für Lerner des Deutschen. Heidelberg: Julius Groos 1981, 137-148.

THIEL, R., «Würzwörter», Sprachpflege 4 (1962), 71-73.

TORRegrosa, J., Análisis multisistémico de las partículas modales del alemán. Tesis Doctoral. Universidad de Barcelona 2010. http://hdl.handle.net/10803/32037. [03/07/2013].

TORREGrosA, J., «Coestructuración melódico-kinésica de la partícula modal alemana doch», en: CABedo, A. / Aguilar, M. J. / LóPez-NAVArro, E. (eds.), Estudios de lingüística: investigaciones, propuestas y aplicaciones. Valencia: Tecnolingüística, SL 2013, 193-203. http://www.uv.es/canea/archivos/Estudios_linguistica_2013.pdf. [09/07/2014].

TORREgrosA, J. / FONT-ROTCHÉS, D., «Melodic patterns of absolute interrogative utterances in northern German in spontaneous speech», Linguistics, en prensa.

UlRICH, M., «Personalpronomina als Abtönungspartikeln?», en: WEYDT, H. (ed.), Sprechen mit Partikeln. Berlín: Walter de Gruyter 1989, 39-46.

VON DER GABElEntZ, G., Die Sprachwissenschaft, ihre Aufgaben, Methoden und bisherigen Ergebnisse. 2a ed., 1969. Tübingen: Narr 1901.

Vural, S., Der Partikelgebrauch im heutigen Deutsch und im heutigen Türkisch. Eine kontrastive Untersuchung. Tesis Doctoral. Universität Mannheim 2000. http://dnb.info/960977287. [07/12/2013].

Wegener, H., «Eine modalpartikel besonderer Art: Der Dativus Ethicus», en: WeYdT, H. (ed.), Sprechen mit Partikeln. Berlín: Walter de Gruyter 1989, 56-73.

Werner, A., Deutsche Modalpartikeln im Kontrast zum Japanischen. Tesis Doctoral. Universität Siegen 1998. http://d-nb.info/958556156. [07/12/2013].

WeStHEIDE, H., «Zur ethnographischen Beschreibung von Partikelfunktionen am Beispiel Niederländisch-Deutsch», en: WEYDT, H. (ed.), Sprechen mit Partikeln. Berlín: Walter de Gruyter 1989, 293-302.

WEYDT, H., Abtönungspartikel. Die deutschen Modalwörter und ihre französischen Entsprechungen. Bad Homburg: Gehlen 1969. 